\title{
Effect on the Limit of Stability of the Lowered Center of Mass With a Weight Belt
}

\author{
Jimmy Phan'1, DPT, Kaylen Wakumoto ${ }^{1}$, DPT, Jeffrey Chen¹, DPT, Woochol Joseph Choi², PT, PhD
}

${ }^{1}$ Department of Physical Therapy, Chapman University, Irvine, CA, USA, ${ }^{2}$ Injury Prevention and Biomechanics Laboratory, Department of Physical Therapy, Yonsei University, Wonju, Korea

\author{
Article Info \\ Received January 30, 2020 \\ Revised March 31, 2020 \\ Accepted April 24, 2020 \\ Corresponding Author \\ Woochol Joseph Choi \\ E-mail:wcjchoi@yonsei.ac.kr \\ https://orcid.org/0000-0002-6623-3806
}

\section{Key Words}

Balance

Center of mass

Limit of stability

Weight belt
Background: The consequences of falls are often debilitating, and prevention is important. In theory, the lower the center of mass (COM), the greater postural stability during standing, and a weight belt at the waist level may help to lower the COM and improve the standing balance.

Objects: We examined how the limit of stability (LOS) was affected by the lowered center of mass with the weight belt.

Methods: Twenty healthy individuals participated in the LOS test. After calculating each participant's COM, a weight belt was fastened ten centimeters below the COM. Trials were acquired with five weight belt conditions: $0 \%, 2 \%, 4 \%, 6 \%$, and $8 \%$ of body weight. Outcome measures included reaction time, movement velocity, endpoint excursion, maximum excursion, and directional control in 4 cardinal moving directions.

Results: None of our outcome variables were associated with a weight belt ( $p>0.075)$, but all of them were associated with moving direction $(p<0.01)$. On average, movement velocity of the COM and maximum excursion were $31 \%$ and $18 \%$ greater, respectively, in mediolateral than anteroposterior direction ( $5.4 \%$ vs. $4.1 \%$; $97.5 \%$ vs. $82.6 \%)$.

Conclusion: Our results suggest that postural stability was not affected by the weightinduced lowered COM, informing the development and improvement of balance training strategies.

\section{INTRODUCTION}

Fall-related injuries are a big health problem. In France, 11\% of women aged over 75 experienced hip fractures die within a year, and the mortality rate becomes 5 times greater when compared to older women who did not have any fractures [1]. In the US, about $20 \%$ of older adults fractured their hip die within a year, and 50\% have permanent disabilities requiring assistive devices for their mobility for the rest of their life [2]. Approximately, 150,000 hip fractures occur annually, and associated health care cost is about 15 billion dollars in the US [3-5]. In South Korea, 14\% and 25\% of older men and women aged over 65, respectively, experience injurious falls each year, and 1,494 older adults died due to falls in 2017 [6]. Among individuals who had a hip fracture, $20 \%$ die within two years and $25 \%$ become dependent in outdoor activities [7]. Furthermore, the fall death rate is substantially higher in older adults aged over $90(91.0$ per 100,000) than aged between 65-69 (11.8 per 100,000) [6].

While efforts have been made to address this issue, it does not seem to be solved yet, and the fall death rate of individuals over 65 have continuously increased over the past decade [8]. As individuals age, they develop an increased risk of falling due to natural changes in the body, activity level, and postural stability. A simple but effective balance training method that might slow down the progress of age-related changes, or improve one's balance, especially for frail population, may help to reduce the incidence of falls and associated injuries in older adults.

A recent study theorized that for every $1 \%$ rise in the height of the center of mass (COM) (i.e., $1.7 \mathrm{~cm}$ increase for an individual who is $170 \mathrm{~cm}$ tall), there is a $37 \%$ increase in the risk of falls (i.e., the odds ratio for falls $=1.37$ ) [9]. Another study has shown that subjects who wore an $8 \mathrm{lb}$ cervical halo performed 
more poorly in single-legged stance and functional reach tests than a control group who did not wear a halo, suggesting that the halo raised the COM and decreased performance on balance tests [10]. These findings suggest that the raised COM challenges one's balance, but this fact could be utilized as a good training concept to improve balance for individuals at high risk of falls. From a mechanical perspective, on the other hand, postural stability increases with lowered COM as a greater angle is needed to cause instability and a smaller recovery torque is required to regain balance [11,12], and could be used as training concepts for the development of interventions for high risk fallers. However, this mechanical notion should be tested in humans prior to use.

Against this background, we have conducted the limit of stability (LOS) tests with healthy adults while lowering the COM with a weight belt, in order to examine how the weight-belt induced lowered COM affects balance, and how benefits of the weight belt, if exists, is influenced by the direction of the body sway. We hypothesized that the postural stability improves as the height of COM decreases with a weight belt, and the effect depends on the direction of body sway.

\section{MATERIALS AND METHODS}

\section{Subjects}

Twenty healthy individuals (males 7 and females 13) of mean age 26 (standard deviation $[S D]=2.43$ ) participated in the LOS test. Average body mass and height were $64.9(\mathrm{SD}=9.0) \mathrm{kg}$ and $165.5(\mathrm{SD}=8.0) \mathrm{cm}$, respectively. Exclusion criteria included inability to speak and understand English, visual deficits, vestibular dysfunctions, inability to bear weight on both legs, and current musculoskeletal injuries. The study protocol was approved by the Institutional Review Board at Chapman University (approval No. 1415H143), and all participants agreed to participate by providing a written informed consent form.

\section{Equipment}

The SMART Balance Master (Natus, San Carlos, CA, USA) was used to obtain quantitative measurements of the Limits of Stability test. The apparatus included a computer, printer, harness, and display monitor. The All Pro Water Walker Weighted Belt with adjustable weights in 5.75 ounces $(0.16 \mathrm{~kg})$ increments (All Pro Exercise Products, Hillsborough, NJ, USA) was also used to alter participant's COM.

A LOS test is an evidence-supported, reliable clinical tool for measuring one's postural stability, and numerous research studies have used the LOS to compare balance in young and older adults, fallers and non-fallers, and individuals with Parkinson's disease and brain injuries [13-19]. The LOS test is a balance test, where participants are instructed to move a cursor that indicates the center of pressure (COP) over the base of support (BOS) as indicated by a center box on the monitor, towards a target positioned near the boundary of the BOS and hold that position for 5 seconds. Changes of COP over time
(Eqn 1) $b m_{b} g=2 R_{1}$

(Eqn 2) $b m_{b} g+x_{C M} m_{h} g=2 R_{2}$

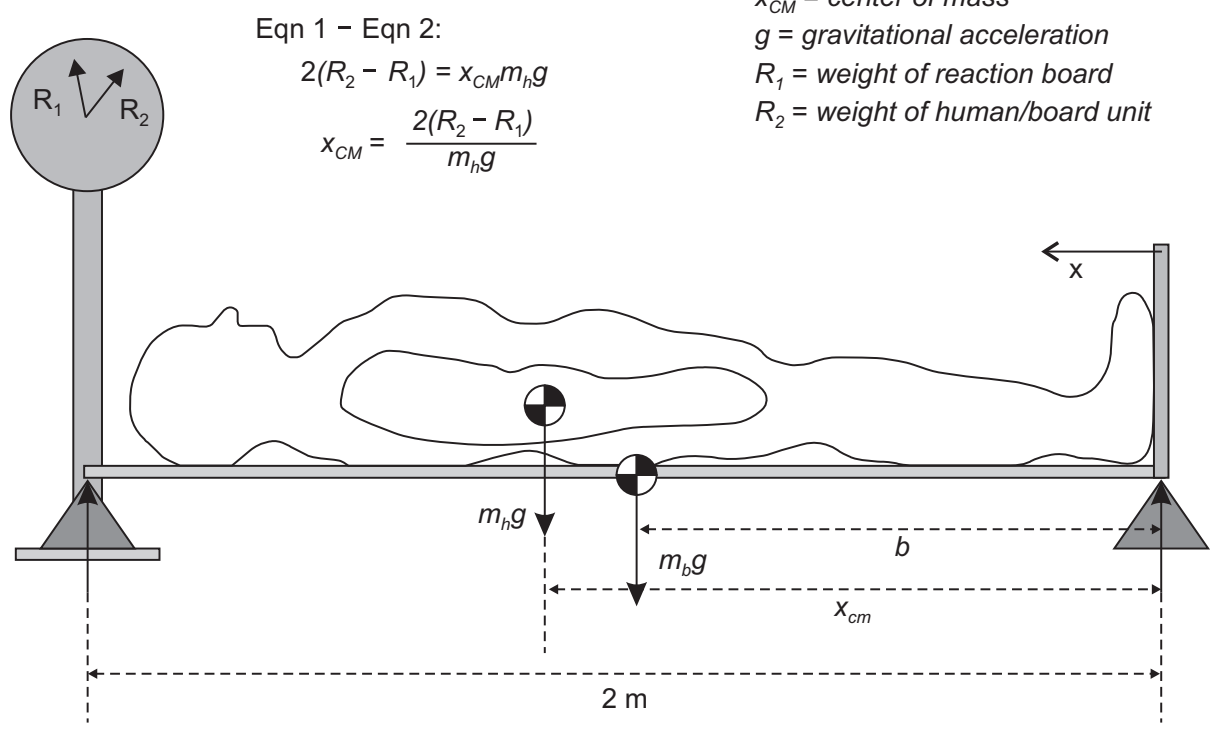

where, $m_{h}=$ human body mass

$m_{b}=$ reaction board mass

$x_{C M}=$ center of mass

$g=$ gravitational acceleration

$R_{1}=$ weight of reaction board

$R_{2}=$ weight of human/board unit
Figure 1. Measurement of the center of mass. 
are used to produce five outcome variables, including reaction time, movement velocity, endpoint excursion, maximum excursion, and directional control, which are used to assess one's postural stability during movement.

\section{Height of Center of Mass}

Prior to performing the Limits of Stability test, the participant's center of mass was determined with a reaction board method [20]. Conditions of rest of a reaction board and a board/human unit follow equations 1 and 2, respectively (Figure 1). By eliminating the term, the height of the center of mass $\left(\chi_{\mathrm{CM}}\right)$ was determined.

\section{Experimental Protocol}

A weight belt was fastened at the waist so the superior border of the belt locates $10 \mathrm{~cm}$ below the COM. While a weight belt at the knees or ankles might be more effective in lowering the COM, it may increase the risk of a fall during activities as well (i.e., bump each other during walking), and the practical use of the belt is limited. Therefore, we decided to apply the belt at the waist level. To perform the LOS tests, participants stepped into the Balance Master, and stood with their bare feet positioned in the center of the force plate, as detected by the machine. During trials, participants were instructed to position a cursor that indicates the center of gravity over the base of support as indicated by a center box on the monitor, towards a target positioned in 4 cardinal directions (right, left, front or back) and hold that position for 5 seconds (Figure 2). All participants initiated this movement immediately following an auditory cue from the machine. Trials were also acquired with five weight belt conditions: $0 \%, 2 \%, 4 \%, 6 \%$, and $8 \%$ of the participant's body weight, where multiple 5.75 ounces weights were attached and evenly distributed on the weight belt to increase the weight of the belt (thereby to lower the COM) during a task. The maximum weight ( $8 \%$ of body weight) was determined based on pilot tests, where participants were able to complete the LOS test with no adverse effects (i.e., soreness, pain). The order of presentation of moving direction and weight condition was randomized.

\section{Data Analysis}

Outcome variables included reaction time (time in seconds between the command to move and the participant's first movement), movement velocity (an average speed of COM movement [i.e., leaning angle] in degrees per second), endpoint excursion (displacement of the first movement towards the target, expressed as a percentage of maximum LOS distance. The endpoint is considered to be the point at which the initial movement toward the target ceases), maximum excursion (maximum displacement achieved during the trial), and directional control (a comparison of the amount of movement in the intended direction [towards the target] to the amount of extraneous movement [away from the target]) for each of 4 cardinal directions (mediolateral and anteroposterior).

For statistical analyses, two-way repeated measures ANOVA was used to test if the outcome variables were associated with the weight belt condition (5 levels) and moving direction (4 levels). When results indicated a significant effect, a pair-wise comparison was conducted using a Bonferroni correction. All analyses were conducted with SPSS version 20 (IBM Co., Ar-
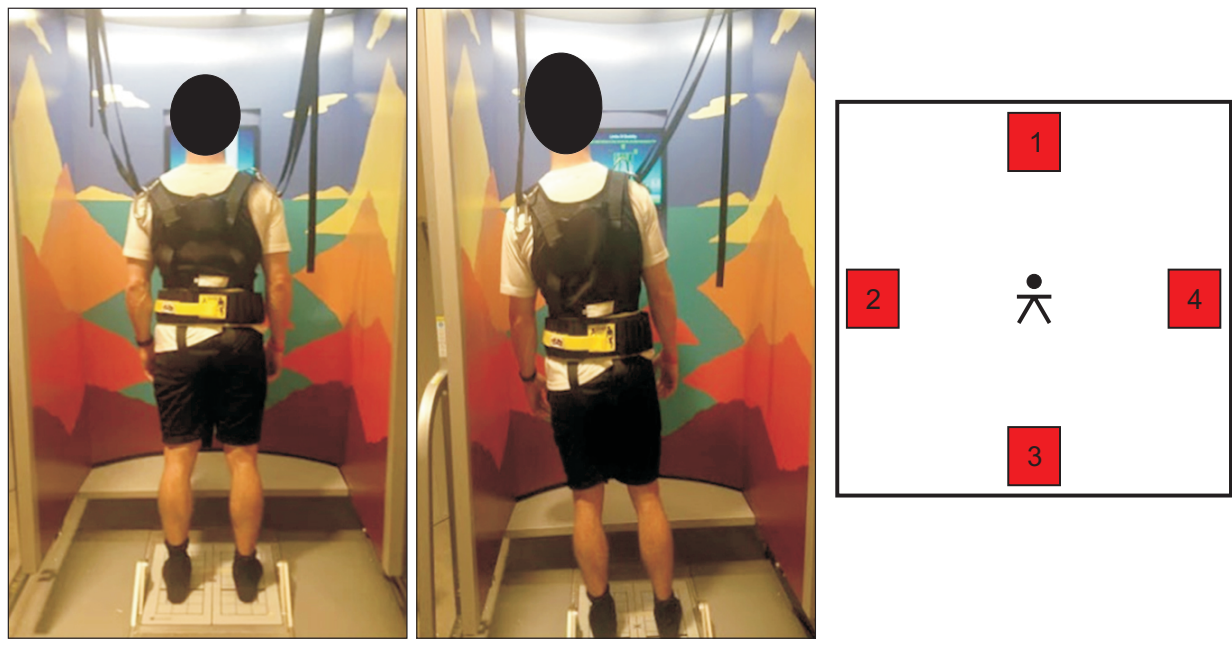

Figure 2. A participant standing with harness, moving toward a target on his left. Locations of targets and the center of mass of the participant are displayed through a monitor. 
monk, NY, USA) using a significance level of alpha $=0.05$.

\section{RESULTS}

Mauchly's test indicated that sphericity has not been violated for most cases in each outcome variable ( $p>0.05)$. When it was violated with an epsilon value less or greater than 0.75 , we interpreted Greenhouse-Geisser or Huynh-Feldt correction, respectively, in the tests of Within-Subjects Effects.

None of our outcome variables were associated with a weight belt $(p>0.075)$, but all of them were associated with moving direction $(\mathrm{p}<0.01)$ (Table 1, Figure 3). On average, movement velocity of the COM (degrees per second) and maximum excursion (maximum displacement as a percentage of maximum LOS distance) were $31 \%$ and $18 \%$ greater, respectively, in mediolateral than anteroposterior direction $\left(5.4^{\circ} / \mathrm{s}\right.$ vs. $4.1 \%$ s; $97.5 \%$ vs. $82.6 \%$ ). Furthermore, there was no interaction between the weighted belt and moving direction ( $p>0.31)$.

\section{DISCUSSION}

A goal of this study was to examine how postural stability is affected by a weight-belt induced lowered COM, and discuss potential benefits of the weight belt to improve balance for individuals at high risk of falls. We first confirmed that the COM lowered up to $8 \mathrm{~cm}$ due to the weight belt (Table 2). However, the weight-belt induced lowered COM did not affect postural stability. This is in contrast to participants' impression acquired from interview after experiments, where they all re-

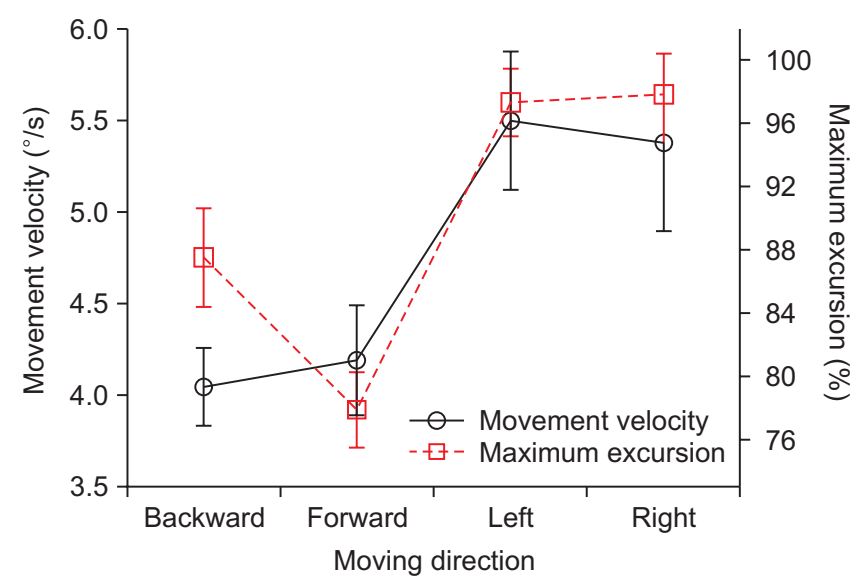

Figure 3. Effect of moving direction on movement velocity and maximum excursion. Participants moved their center of mass further with faster speed in mediolateral than anterioposterior direction.

Table 1. Average values of outcome variables (with SD shown in parentheses)

\begin{tabular}{|c|c|c|c|c|c|c|}
\hline $\begin{array}{c}\text { Belt weight } \\
\text { (\% of body weight) }\end{array}$ & Moving direction & $\begin{array}{l}\text { Reaction time } \\
\text { (s) }\end{array}$ & $\begin{array}{l}\text { Movement velocity } \\
\qquad(\%)\end{array}$ & $\begin{array}{l}\text { Endpoint excursion } \\
\qquad(\%)\end{array}$ & $\begin{array}{c}\text { Maximum excursion } \\
{[\%]}\end{array}$ & $\begin{array}{c}\text { Directional control } \\
\qquad \%]\end{array}$ \\
\hline \multirow[t]{5}{*}{$0 \%$} & Backward & $0.682(0.152)$ & $3.9(1.0)$ & 75.7 (18.7) & 86.2 (15.9) & $87.2(5.0)$ \\
\hline & Forward & $0.766(0.201)$ & $4.1(1.2)$ & $72.0(11.0)$ & $77.8(12.1)$ & $89.5(3.0)$ \\
\hline & Left & $0.791(0.196)$ & $5.5(1.8)$ & $88.3(12.1)$ & $96.3(10.0)$ & $88.1(5.2)$ \\
\hline & Right & $0.781(0.232)$ & $5.2(2.4)$ & 90.5 (12.0) & $99.1(12.6)$ & $87.4(5.0)$ \\
\hline & Mean & 0.755 (0.195) & $4.7(1.6)$ & $81.6(13.4)$ & $89.8(12.6)$ & $88.0(4.6)$ \\
\hline \multirow[t]{5}{*}{$2 \%$} & Backward & $0.715(0.186)$ & $4.3(1.0)$ & 81.1 (13.8) & $88.6(14.1)$ & $87.1(4.2)$ \\
\hline & Forward & $0.817(0.219)$ & $4.2(1.6)$ & $70.7(10.1)$ & $77.3(11.7)$ & 89.8 (3.3) \\
\hline & Left & 0.762 (0.193) & $5.4(1.9)$ & $93.3(10.6)$ & 99.7 (10.5) & $88.1(3.1)$ \\
\hline & Right & 0.791 (0.209) & $5.4(2.5)$ & 91.1 (11.2) & $98.1(13.6)$ & $87.5(4.5)$ \\
\hline & Mean & $0.771(0.202)$ & $4.8(1.7)$ & $84.0(11.4)$ & $90.9(12.5)$ & $88.1(3.8)$ \\
\hline \multirow[t]{5}{*}{$4 \%$} & Backward & $0.653(0.165)$ & $4.0(1.2)$ & 80.3 (14.8) & 87.8 (14.8) & $86.1(6.7)$ \\
\hline & Forward & $0.840(0.227)$ & $4.4(1.6)$ & $69.6(11.4)$ & 78.1 (11.3) & $88.5(3.6)$ \\
\hline & Left & $0.756(0.221)$ & $5.4(1.6)$ & 90.9 (11.0) & $98.9(10.3)$ & $86.5(5.1)$ \\
\hline & Right & $0.775(0.229)$ & $5.6(2.3)$ & $92.3(11.1)$ & $99.2(10.7)$ & $86.5(5.1)$ \\
\hline & Mean & $0.756(0.210)$ & $4.8(1.7)$ & $83.3(12.1)$ & $91.0(11.8)$ & $86.9(5.1)$ \\
\hline \multirow[t]{5}{*}{$6 \%$} & Backward & $0.732(0.227)$ & $3.9(1.1)$ & 78.3 (12.9) & 88.0 (14.8) & $87.1(2.9)$ \\
\hline & Forward & $0.862(0.288)$ & $4.1(1.5)$ & 72.7 (12.2) & $78.7(12.6)$ & $89.5(2.4)$ \\
\hline & Left & $0.790(0.241)$ & $5.7(2.2)$ & $86.1(15.0)$ & 96.7 (11.5) & $88.1(2.8)$ \\
\hline & Right & $0.875(0.254)$ & $5.2(2.1)$ & $89.2(12.0)$ & $96.2(13.0)$ & 88.8 (3.7) \\
\hline & Mean & $0.815(0.252)$ & $4.7(1.7)$ & 81.6 (13.0) & 89.9 (13.0) & $88.4(2.9)$ \\
\hline \multirow[t]{5}{*}{$8 \%$} & Backward & $0.710(0.172)$ & $4.1(1.3)$ & $78.8(15.1)$ & $87.1(16.1)$ & $86.7(5.4)$ \\
\hline & Forward & $0.912(0.284)$ & $4.2(1.7)$ & $69.9(11.1)$ & 77.8 (11.8) & $90.0(2.9)$ \\
\hline & Left & $0.845(0.230)$ & $5.6(2.0)$ & $87.6(13.7)$ & 95.2 (12.8) & $89.2(3.0)$ \\
\hline & Right & $0.875(0.246)$ & $5.5(2.1)$ & $89.7(15.6)$ & $96.8(14.4)$ & 88.2 (3.8) \\
\hline & Mean & $0.835(0.233)$ & $4.8(1.8)$ & 81.5 (13.9) & 89.2 (13.8) & 88.5 (3.8) \\
\hline
\end{tabular}


Table 2. Changes of the height of center of mass with a weight belt

\begin{tabular}{|c|c|c|c|c|c|c|}
\hline \multirow{2}{*}{ Participant } & \multirow{2}{*}{ Body height $(\mathrm{cm})$} & \multirow{2}{*}{ Height of $\mathrm{COM}(\mathrm{cm})$} & \multicolumn{4}{|c|}{ Lowered COM with a weight belt $(\mathrm{cm})$} \\
\hline & & & $2 \%$ & $4 \%$ & $6 \%$ & $8 \%$ \\
\hline 1 & 173.5 & 114.7 & 112.5 & 110.3 & 108.2 & 106.2 \\
\hline 2 & 163.8 & 106.5 & 104.4 & 102.4 & 100.5 & 98.6 \\
\hline 3 & 158.5 & 96.5 & 94.6 & 92.8 & 91.0 & 89.4 \\
\hline 4 & 153.0 & 99.0 & 97.1 & 95.2 & 93.4 & 91.7 \\
\hline 5 & 181.0 & 115.9 & 113.6 & 111.4 & 109.3 & 107.3 \\
\hline 6 & 173.5 & 110.3 & 108.1 & 106.1 & 104.1 & 102.1 \\
\hline 7 & 153.0 & 98.2 & 96.3 & 94.4 & 92.7 & 90.9 \\
\hline 8 & 159.5 & 103.3 & 101.3 & 99.3 & 97.5 & 95.7 \\
\hline 9 & 170.5 & 108.0 & 105.9 & 103.9 & 101.9 & 100.0 \\
\hline 10 & 170.0 & 106.0 & 103.9 & 101.9 & 100.0 & 98.2 \\
\hline 11 & 162.0 & 99.8 & 97.8 & 96.0 & 94.2 & 92.4 \\
\hline 12 & 172.0 & 109.6 & 107.5 & 105.4 & 103.4 & 101.5 \\
\hline 13 & 160.5 & 111.3 & 109.1 & 107.0 & 105.0 & 103.1 \\
\hline 14 & 162.5 & 104.8 & 102.7 & 100.8 & 98.9 & 97.0 \\
\hline 15 & 176.0 & 115.8 & 113.5 & 111.4 & 109.3 & 107.2 \\
\hline 16 & 163.0 & 106.4 & 104.3 & 102.3 & 100.4 & 98.5 \\
\hline 17 & 163.0 & 106.4 & 104.3 & 102.3 & 100.4 & 98.5 \\
\hline 18 & 161.0 & 102.0 & 100.0 & 98.1 & 96.2 & 94.5 \\
\hline 19 & 176.5 & 116.9 & 114.6 & 112.4 & 110.3 & 108.3 \\
\hline 20 & 157.5 & 101.7 & 99.7 & 97.8 & 96.0 & 94.2 \\
\hline
\end{tabular}

COM, center of mass.

ported increased stability during movement. Biomechanically, postural stability requires that the vertical line passing through the COM locates within the base of support formed by the feet. Furthermore, postural stability is improved as the height of the COM decreases. This is due, in part, to that a greater angle (perturbation) is required to cause instability and a smaller recovery torque is needed to recover balance if the height of the COM is decreased [11,12]. However, our data suggest that postural stability would not be improved when individuals' COM is lowered with a weight belt. While difficult to discuss what caused this discrepancy between the experimental observations and theoretical predictions, the difference might come from balance strategies (hip vs. angle strategies) that participants employed during a task. The theoretical predictions are based on a single degree of freedom inverted pendulum model, where a torque at the ankle joint (no knee and hip joints) is the only source of a force that controls balance during standing. Whereas, our participants were free in selecting balance strategies during task, and might use hip or ankle strategy, or both.

Another goal of this study was to examine how benefits of a weight-induced lowered COM during standing if exist, are affected by the direction of the body sway. As discussed, our results suggest that there are no potential benefits of the weightinduced lowered COM in balance control during LOS tests (main effect). Therefore, it is pointless to discuss an interaction effect, which was not statistically significant anyway $(\mathrm{p}>0.31)$. However, we found that the balance was affected by moving direction, suggesting the mediolateral movement would be more stable than anteriolateral direction during standing. This makes sense biomechanically as a mediolaterally wider shape of the base of support during standing provides more resistance to lateral forces, allowing more efficient control of the COM under circumstances of mediolatearal perturbations. In reality, however, a large number of falls are initiated sideways (mediolaterally), and end up with impacting the lateral side of the body (hip, thigh, trunk, head), particularly in older adults $[21,22]$. Balance is largely affected by neuromuscular responses (i.e., reaction time, muscle strength, vision), which likely decline with age and comorbidities (i.e., hypotension, sarcopenia) commonly observed in older adults. Therefore, aging effects (which was not part of this study) should be considered when discussing mechanics of balance and falling in older adults.

Our results should be interpreted in light of several limitations. First, while the height of COM of participants was believed to be lowered by a weight belt, we have not monitored the COM changes induced by the weight belt. Second, we had to include healthy young individuals only in our experiments, 
in order to test with greater weight (up to $8 \%$ of body weight) as possible, which may cause adverse events (fatigue, dizziness, falls) in older adults. Third, while the LOS is considered to be a good clinical tool to assess one's postural stability, tasks only involved movements while standing, which does not cover all circumstances of falls. Collectively, future studies involving measures of kinematics to monitor COM changes with a lighter weight belt in older adults under various situations (i.e., turning, transferring, walking), are warranted to continue to explore potential benefits of weight belt-induced lowered COM in postural stability. However, the weight-belt induced lowered COM may cause discomfort that limits normal movements and activities. Therefore, potential negative effects should also be examined along with the benefits.

\section{CONCLUSIONS}

A simple but effective training strategy to improve one's postural stability should be appreciated by clinicians and individuals at high risk of falls. In contrast to theoretical predictions, the potential benefits of a weight belt-induced lowered COM were not found in healthy adults during LOS tests. Therefore, balance training methods involving the weight belt may not provide additional help to improve one's balance.

\section{CONFLICTS OF INTEREST}

No potential conflict of interest relevant to this article was reported.

\section{AUTHOR CONTRIBUTIONS}

Conceptualization: JP, KW, JC, WJC. Data collection: JP, KW, JC. Data analysis: JP, KW, JC, WJC. Supervision: WJC. Writing original draft: JP, KW, JC, WJC. Writing - review \& editing: JP, KW, JC, WJC.

\section{REFERENCES}

1. Empana JP, Dargent-Molina P, Bréart G; EPIDOS Group. Effect of hip fracture on mortality in elderly women: the EPIDOS prospective study. J Am Geriatr Soc 2004;52(5):685-90.

2. Wolinsky FD, Fitzgerald JF, Stump TE. The effect of hip fracture on mortality, hospitalization, and functional status: a pro- spective study. Am J Public Health 1997;87(3):398-403.

3. Brauer CA, Coca-Perraillon M, Cutler DM, Rosen AB. Incidence and mortality of hip fractures in the United States. JAMA 2009;302(14):1573-9.

4. Burge R, Dawson-Hughes B, Solomon DH, Wong JB, King A, Tosteson A. Incidence and economic burden of osteoporosisrelated fractures in the United States, 2005-2025. J Bone Miner Res 2007;22(3):465-75.

5. Gillespie WJ. Extracts from "clinical evidence": hip fracture. BMJ 2001;322(7292):968-75.

6. Statistics Korea. The fall experience of the elderly adults. Statistics Korea [Internet]. Daejeon: 2018 Dec [cited 2020 Jan 30]. Available from: http://kosis.kr/statHtml/statHtml.do?orgId= 117\&tblId=DT_117071_050\&conn_path=I2.

7. Lee SR, Kim SR, Chung KH, Ko DO, Cho SH, Ha YC, et al. Mortality and activity after hip fracture: a prospective study. J Korean Orthop Assoc 2005;40(4):423-7.

8. Burns E, Kakara R. Deaths from falls among persons aged $\geq 65$ years - United States, 2007-2016. MMWR Morb Mortal Wkly Rep 2018;67(18):509-14.

9. Almeida CW, Castro CH, Pedreira PG, Heymann RE, Szejnfeld VL. Percentage height of center of mass is associated with the risk of falls among elderly women: a case-control study. Gait Posture 2011;34(2):208-12.

10. Richardson JK, Ross AD, Riley B, Rhodes RL. Halo vest effect on balance. Arch Phys Med Rehabil 2000;81(3):255-7.

11. Bridger RS. Introduction to human factors and ergonomics. 4th ed. Boca Raton: CRC Press; 2018;108-10.

12. Wan FKW, Yick KL, Yu WWM. Effects of heel height and highheel experience on foot stability during quiet standing. Gait Posture 2019;68:252-7.

13. Boulgarides LK, McGinty SM, Willett JA, Barnes CW. Use of clinical and impairment-based tests to predict falls by community-dwelling older adults. Phys Ther 2003;83(4):328-39.

14. Brauer SG, Burns YR, Galley P. A prospective study of laboratory and clinical measures of postural stability to predict community-dwelling fallers. J Gerontol A Biol Sci Med Sci 2000;55(8):M469-76.

15. Clark S, Rose DJ. Evaluation of dynamic balance among community-dwelling older adult fallers: a generalizability study of the limits of stability test. Arch Phys Med Rehabil 2001;82(4):468-74.

16. Mancini M, Rocchi L, Horak FB, Chiari L. Effects of Parkinson's disease and levodopa on functional limits of stability. Clin Bio- 
mech (Bristol, Avon) 2008;23(4):450-8.

17. Melzer I, Benjuya N, Kaplanski J. Postural stability in the elderly: a comparison between fallers and non-fallers. Age Ageing 2004;33(6):602-7.

18. Newstead AH, Hinman MR, Tomberlin JA. Reliability of the Berg Balance Scale and balance master limits of stability tests for individuals with brain injury. J Neurol Phys Ther 2005;29(1):18-23.

19. Wallmann HW. Comparison of elderly nonfallers and fallers on performance measures of functional reach, sensory organization, and limits of stability. J Gerontol A Biol Sci Med Sci 2001;56(9):M580-3
20. Özkaya N, Nordin M, Goldsheyder D, Leger D. Fundamentals of biomechanics: equilibrium, motion, and deformation. 3rd ed. New York: Springer; 2012;53-4.

21. Robinovitch SN, Feldman F, Yang Y, Schonnop R, Leung PM, Sarraf T, et al. Video capture of the circumstances of falls in elderly people residing in long-term care: an observational study. Lancet 2013;381 (9860):47-54.

22. Schonnop R, Yang Y, Feldman F, Robinson E, Loughin M, Robinovitch SN. Prevalence of and factors associated with head impact during falls in older adults in long-term care. CMAJ 2013;185(17):E803-10. 